\title{
Bernstein operator of rough J-core of triple sequences
}

\author{
M. Kemal Ozdemir ${ }^{1, *}$, Ayhan $\mathrm{Esi}^{2, * *}$, and Ayten $\mathrm{Esi}^{2, * * *}$ \\ ${ }^{1}$ Department of Mathematics, İnönü University, 44280, Malatya, Turkey \\ ${ }^{2}$ Department of Mathematics, Adıyaman University, 02040, Adıyaman, Turkey
}

\begin{abstract}
We introduce and study some basic properties of Bernstein-Stancu polynomials of rough J-convergent of triple sequence spaces and also study the set of all Bernstein-Stancu polynomials of rough J-limits of a triple sequence spaces and relation between analytic ness and Bernstein-Stancu polynomials of rough J-core of a triple sequence spaces.
\end{abstract}

\section{Introduction}

The notion of rough convergence has firstly been presented by Phu [10-12] in finite dimensional normed spaces. The author also illustrated that this set $L I M_{x}^{r}$ is closed, convex and bounded; and he at the same time proposed the concept about the rough Cauchy sequence. The author again examined the connections among rough convergence and the other types of convergence and the dependence for $L I M_{x}^{r}$ on the roughness having degree of $r$. Aytar [1] investigated rough statistical convergence and described rough statistical limit points set for a given sequence and had two important criteria about statistical convergence related to the set and proved the fact that the set is convex and closed. Meanwhile, Aytar [2] investigated that the $r$-limit set of the sequence equals to the intersection of those sets and that $r$-core of the sequence equals to the union of those sets. Dundar and Cakan [9] investigated rough ideal convergence and defined the set of rough ideal limit points of a sequence. The concept of $J$-convergence of a triple sequence spaces depended on the very structure for the ideal $\mathcal{J}$ of the subsets of $\mathbb{N}^{3}$, where $\mathbb{N}$ denotes the natural numbers, is an expected result of the concept of convergence and statistical convergence.

In the present manuscript, we investigate several fundamental characteristics for rough J-convergence of a triple sequence spaces in three dimensional matrix spaces. We analyze the set of all rough J-limits consisting of a triple sequence spaces and at the same time the connection among analytic ness and rough J-core of a triple sequence spaces.

Let us assume that $K$ is a subset consisting of the set consisting of positive integer numbers $\mathbb{N}^{3}$ and let us assume this set $K_{i k \ell}=\{(m, n, k) \in K: m \leq i, n \leq j, k \leq \ell\}$. Under these assumptions, the natural density for $K$ can be denoted by $\delta(K)=\lim _{i, j, \ell \rightarrow \infty} \frac{\left|K_{i j \ell}\right|}{i j \ell}$, where $\left|K_{i j \ell}\right|$ stands for the number of elements in $K_{i j \ell}$.

First applied the notion of $(p, q)$-calculus in approximation theory and put forward the $(p, q)$-analogue of Bernstein operators. Later, depending on $(p, q)$-integers, several approximation results for Bernstein-Stancu, Bernstein-Kantorovich, $(p, q)$-Lorentz, BernsteinSchurer, Bleimann, Butzer and Hahn operators etc.

\footnotetext{
*e-mail: kozdemir73@gmail.com

**e-mail: aesi23@hotmail.com

***e-mail: aytenesi@yahoo.com
} 
In recent years, Khalid et al. have presented a very nice application using computer-aided geometric design and also applied those Bernstein basis to construct $(p, q)$-Bezier curves and surfaces depending upon $(p, q)$-integers, being a step other generalization of $q$-Bezier surfaces and curves.

Motivated by the above mentioned work on $(p, q)$-approximation and its application, in this paper we study statistical approximation properties of BernsteinStancu Operators depending on $(p, q)$-integers. Now we are going to remember several fundamental descriptions related to $(p, q)$-integers. For any $u, v, w \in \mathbb{N}$, the $(p, q)$-integer $[u v w]_{p, q}$ is described as

$$
[0]_{p, q}:=0 \text { and }[u v w]_{p, q}=\frac{p^{u v w}-q^{u v w}}{p-q} \text { if } u, v, w \geq 1,
$$

in which $0<q<p \leq 1$. The $(p, q)$-factorial is described as

$$
[0]_{p, q} !:=1 \text { and }[u v w] !_{p, q}=[1]_{p, q}[2]_{p, q} \cdots[u v w]_{p, q} \text { if } u, v, w \geq 1 .
$$

We can describe the $(p, q)$-binomial coefficient as

$$
\left(\begin{array}{l}
u \\
m
\end{array}\right)\left(\begin{array}{l}
v \\
n
\end{array}\right)\left(\begin{array}{l}
w \\
k
\end{array}\right)_{p, q}=\frac{[u] !_{p, q}}{[m] !_{p, q}[u-m] !_{p, q}} \frac{[v] !_{p, q}}{[n] !_{p, q}[v-n] !_{p, q}} \frac{[w] !_{p, q}}{[k] !_{p, q}[w-k] !_{p, q}}
$$

for all $u, v, w, m, n, k \in \mathbb{N}$ with $(u, v, w) \geq(m, n, k)$.

The equality about $(p, q)$-binomial expansion can be given as

$$
\begin{gathered}
(a x+b y)_{p, q}^{u v w}=\sum_{m=0}^{u} \sum_{n=0}^{v} \sum_{k=0}^{w} p^{\frac{(u-m)(u-m-1)+(v-n)(v-n-1)+(w-k)(w-k-1)}{2}} q^{\frac{m(m-1)+n(n-1)+k(k-1)}{2}} . \\
\left(\begin{array}{l}
u \\
m
\end{array}\right)\left(\begin{array}{l}
v \\
n
\end{array}\right)\left(\begin{array}{l}
w \\
k
\end{array}\right)_{p, q} a^{(u-m)+(v-n)+(w-k)} b^{m+n+k} x^{(u-m)+(v-n)+(w-k)} y^{m+n+k}, \\
(x+y)_{p, q}^{u v w}=(x+y)(p x+q y)\left(p^{2} x+q^{2} y\right) \cdots\left(p^{(u-1)+(v-1)+(w-1)} x+q^{(u-1)+(v-1)+(w-1)} y\right), \\
(1-x)_{p, q}^{u v w}=(1-x)(p-q x)\left(p^{2}-q^{2} x\right) \cdots\left(p^{(u-1)+(v-1)+(w-1)}-q^{(u-1)+(v-1)+(w-1)} x\right), \text { and } \\
(x)_{p, q}^{m n k}=x(p x)\left(p^{2} x\right) \cdots\left(p^{(u-1)+(v-1)+(w-1)} x\right)=p^{\frac{m(m-1)+n(n-1)+k(k-1)}{2}} .
\end{gathered}
$$

The Bernstein operator of order $(r, s, t)$ is presented as follows

$$
B_{r s t}(f, x)=\sum_{m=0}^{r} \sum_{n=0}^{s} \sum_{k=0}^{t} f\left(\frac{m n k}{r s t}\right)\left(\begin{array}{l}
r \\
m
\end{array}\right)\left(\begin{array}{l}
s \\
n
\end{array}\right)\left(\begin{array}{l}
t \\
k
\end{array}\right) x^{m+n+k}(1-x)^{(m-r)+(n-s)+(k-t)}
$$

in which $f$ is a continuous (either complex or real valued) function on $[0,1]$.

We can define $(p, q)$-Bernstein operators as

$$
\begin{aligned}
& B_{r s t, p, q}(f, x)=\frac{1}{p^{\frac{r(r-1)+s(s-1)+(t(-))}{2}}} \sum_{m=0}^{r} \sum_{n=0}^{s} \sum_{k=0}^{t}\left(\begin{array}{l}
r \\
m
\end{array}\right)\left(\begin{array}{l}
s \\
n
\end{array}\right)\left(\begin{array}{l}
t \\
k
\end{array}\right) p^{\frac{m(m-1)+n(n-1)+k(k-1)}{2}} x^{m+n+k} . \\
& \prod_{u_{1}=0}^{(r-m-1)}\left(p^{u_{1}}-q^{u_{1}} x\right) \prod_{u_{2}=0}^{(s-n-1)}\left(p^{u_{2}}-q^{u_{2}} x\right) \prod_{u_{3}=0}^{(t-k-1)}\left(p^{u_{3}}-q^{u_{3}} x\right) . \\
& f\left(\frac{p^{(r-m)}[m]_{p, q}+p^{(s-n)}[n]_{p, q} p^{(t-k)}[k]_{p, q}}{[r]_{p, q}+[s]_{p, q}+[t]_{p, q}}\right), x \in[0,1]
\end{aligned}
$$


Also, we have

$$
\begin{aligned}
(1-x)_{p, q}^{r s t} & =\sum_{m=0}^{r} \sum_{n=0}^{s} \sum_{k=0}^{t}(-1)^{m+n+k} p^{\frac{(r-m)(r-m-1)+(s-n)(s-n-1)+(t-k)(t-k-1)}{2}} q^{\frac{m(m-1)+n(n-1)+k(k-1)}{2}}\left(\begin{array}{l}
r \\
m
\end{array}\right)\left(\begin{array}{l}
s \\
n
\end{array}\right)\left(\begin{array}{l}
t \\
k
\end{array}\right) x^{m+n+k} \\
& S_{r s t, p, q}(f, x)=\frac{1}{p^{\frac{r(r-1)+s(s-1)+(t-1)}{2}}} \sum_{m=0}^{r} \sum_{n=0}^{s} \sum_{k=0}^{t}\left(\begin{array}{l}
r \\
m
\end{array}\right)\left(\begin{array}{l}
s \\
n
\end{array}\right)\left(\begin{array}{l}
t \\
k
\end{array}\right) p^{\frac{m(m-1)+n(n-1)+k(k-1)}{2}} x^{m+n+k} . \\
& \left(\prod_{u_{1}=0}^{r-m-1)}\left(p^{u_{1}}-q^{u_{1}} x\right) \prod_{u_{2}=0}^{(s-n-1)}\left(p^{u_{2}}-q^{u_{2}} x\right) \prod_{u_{3}=0}^{(t-k-1)}\left(p^{u_{3}}-q^{u_{3}} x\right) \cdot\right. \\
& f\left(\frac{p^{(r-m)}[m]_{p, q}+p^{(s-n)}[n]_{p, q} p^{(t-k)}[k]_{p, q}+\eta}{[r]_{p, q}+[s]_{p, q}+[t]_{p, q}+\mu}\right), x \in[0,1]
\end{aligned}
$$

Note that for $\eta=\mu=0,(p, q)$-Bernstein-Stancu operators in Eq. (1.2) reduces into $(p, q)$ Bernstein operators. Also for $p=1,(p, q)$-Bernstein-Stancu operators in Eq. (1.1) result in $q$-Bernstein-Stancu operators.

A triple sequence (either complex or real) may be described as a function $x: \mathbb{N}^{3} \rightarrow \mathbb{R}(\mathbb{C})$, in which the complex, real and natural numbers are denoted by $\mathbb{C}, \mathbb{R}$ and $\mathbb{N}$, respectively. The various types of concepts for triple sequence has been first proposed and examined initially by Datta et al. [7], Debnath et al. [8], Esi et al. [3-6], Sahiner et al. [13, 14], Subramanian et al. [15] and several others.

It is said that a triple sequence $x=\left(x_{m n k}\right)$ is triple analytic when

$$
\sup _{m, n, k}\left|x_{m n k}\right|^{\frac{1}{m+n+k}}<\infty \text {. }
$$

$\Lambda^{3}$ usually denotes all triple analytic sequences space.

\section{Definitions and Preliminaries}

For the rest of the manuscript $\mathbb{R}^{3}$ symbolizes the real three dimensional case having the metric. Take into consideration a triple sequence $x=\left(x_{m n k}\right)$ such that $x_{m n k} \in \mathbb{R}^{3} ; m, n, k \in \mathbb{N}^{3}$. The following definition are obtained:

Definition 2.1 Assume that $f$ is a continuous function on [0,1]. A triple sequence of Bernstein-Stancu polynomials $\left(S_{r s t, p, q}(f, x)\right)$ is statistically convergent to $(f, x)$ and denoted by $S_{r s t, p, q}(f, x) \rightarrow^{s t-\lim x}(f, x)$, when for every $\epsilon>0, d(A(\epsilon))=0$ is obtained, in which $A(\epsilon)=\left\{(m, n, k) \in \mathbb{N}^{3}:\left|S_{r s t, p, q}(f, x)-(f, x)\right| \geq \epsilon\right\}$.

Definition 2.2 Assume that $f$ is any given continuous function on [0,1]. It is said that a triple sequence of Bernstein-Stancu polynomials $\left(S_{r s t, p, q}(f, x)\right)$ is statistically convergent to $(f, x)$ denoted by $S_{r s t, p, q}(f, x) \rightarrow^{s t-\lim x}(f, x)$, providing that the set $\left\{(m, n, k) \in \mathbb{N}^{3}:\left|S_{r s t, p, q}(f, x)-(f, x)\right| \geq \epsilon\right\}$, with natural density zero for each $\epsilon>0$. Under this condition, $(f, x)$ is known as the statistical limit for the sequence of Bernstein-Stancu polynomials.

Definition 2.3 Assume that $f$ is a given continuous function given on [0,1]. It is said that a triple sequence of Bernstein-Stancu polynomials $\left(S_{r s t, p, q}(f, x)\right)$ in a metric space $(X,|.,|$.$) and$ $r \geq 0$ be a real number is $r$-convergent to $(f, x)$, denoted by $S_{r s t, p, q}(f, x) \rightarrow^{r}(f, x)$, if for any $\epsilon>0$ there exists $N_{\epsilon} \in \mathbb{N}^{3}$ such that for all $m, n, k \geq N_{\epsilon}$ we have $\left|S_{r s t, p, q}(f, x)-(f, x)\right|<r+\epsilon$. In this case $S_{r s t, p, q}(f, x)$ is called an r-limit of $(f, x)$. 
Remark 2.1 We consider r-limit set $S_{r s t, p, q}(f, x)$ which is denoted by $\operatorname{LIM}^{r} S_{r s t, p, q}(f, x)$ and is denoted as $\operatorname{LIM}^{r} S_{r s t, p, q}(f, x)=\left\{f: S_{r s t, p, q}(f, x) \rightarrow^{r}(f, x)\right\}$.

Definition 2.4 It is assumed that $f$ is a prescribed continuous function defined on [0,1]. It is said that a triple sequence of Bernstein-Stancu polynomials $\left(S_{r s t, p, q}(f, x)\right)$ is r-convergent if $L_{I M} S_{r s t, p, q}(f, x) \neq \phi$ and $r$ is known as a rough convergence degree of $S_{r s t, p, q}(f, x)$. If $r=0$ then it is ordinary convergence of triple sequence of Bernstein-Stancu polynomials.

Definition 2.5 Assume that $f$ is a continuous function prescribed on $[0,1]$. It is said that $A$ triple sequence of Bernstein polynomials $\left(B_{m n k}(f, x)\right)$ in a metric space $(X,|.,|$.$) and r \geq 0$ is a real number is $r$-statistically convergent to $(f, x)$, symbolized by $B_{m n k}(f, x) \rightarrow^{r-s t_{3}}(f, x)$, when for any $\epsilon>0$ we obtain $d(A(\epsilon))=0$, in which

$$
A(\epsilon)=\left\{(m, n, k) \in \mathbb{N}^{3}:\left|B_{m n k}(f, x)-(f, x)\right| \geq r+\epsilon\right\} .
$$

When this happens, $(f, x)$ is known as r-statistical limit of $B_{m n k}(f, x)$. If $r=0$ then it is ordinary statistical convergent of triple sequence of Bernstein polynomials.

Definition 2.6 It is said that a class $\mathcal{J}$ of subsets consisting of a nonempty set $X$ is an ideal in $X$ providing that (i) $\phi \in \mathcal{J}$, (ii) $A, B \in \mathcal{J} \Rightarrow A \cup B \in \mathcal{J}$, (iii) $A \in \mathcal{J}, B \subset A \Rightarrow B \in \mathcal{J}$. J is known as a non-trivial ideal if $X \notin \mathcal{J}$.

Definition 2.7 Assume that $f$ is a continuous function on $[0,1]$. It is said that a triple sequence of Bernstein polynomials $\left(B_{m n k}(f, x)\right)$ in a metric space $(X,|.,|$.$) and r \geq 0$ be a real number is rough ideal convergent or $r \mathrm{~J}$-convergent to $(f, x)$, denoted by $B_{\text {mnk }}(f, x) \rightarrow^{r \mathrm{~J}}(f, x)$, if for any $\epsilon>0$ we have $\left\{(m, n, k) \in \mathbb{N}^{3}:\left|B_{\text {mnk }}(f, x)-(f, x)\right| \geq r+\epsilon\right\} \in \mathcal{J}$. In this case $\left(B_{\text {mnk }}(f, x)\right)$ is called $r$ J-convergent to $(f, x)$ and a triple sequence of Bernstein polynomials $\left(B_{\text {mnk }}(f, x)\right)$ is called rough J-convergent to $(f, x)$ with $r$ as roughness of degree. If $r=0$ then it is ordinary J-convergent.

Note 2.1 Generally, it is assumed that $f$ is a continuous function on [0,1]. A triple sequence of Bernstein polynomials $\left(B_{m n k}(g, x)\right)$ is not J-convergent in usual sense and $\left|B_{m n k}(f, x)-B_{m n k}(g, x)\right| \leq r$ for all $(m, n, k) \in \mathbb{N}^{3}$ or $\left\{(m, n, k) \in \mathbb{N}^{3}:\left|B_{\text {mnk }}(f, x)-B_{\text {mnk }}(g, x)\right| \geq r\right\} \in \mathcal{J}$ for some $r>0$. Then the triple sequence of Bernstein polynomials $\left(B_{m n k}(f, x)\right)$ is $r$ J-convergent.

Note 2.2 It is clear that $r$ J-limit of a sequence $B_{m n k}(f, x)$ of Bernstein polynomial is not necessarily unique.

Definition 2.8 Let us consider rJ-limit set of $f(x)$, symbolized by J $-\operatorname{LIM}^{r} B_{m n k}(f, x)=$ $\left\{f: B_{\text {mnk }}(f, x) \rightarrow^{r \mathcal{J}}(f, x)\right\}$, then the triple sequence of Bernstein polynomials $\left(B_{\text {mnk }}(f, x)\right)$ is said to be $r \mathrm{~J}$-convergent if J - LIM $M^{r} B_{m n k}(f, x) \neq \phi$ and $r$ is known as a rough J-convergence having degree of $B_{m n k}(f, x)$.

Definition 2.9 Assume that $f$ is a continuous function on [0,1]. It is said that a triple sequence of Bernstein polynomials $\left(B_{m n k}(f, x)\right)$ is J-analytic if there exists a real number $M>0$ satisfying

$$
\left\{(m, n, k) \in \mathbb{N}^{3}:\left|B_{m n k}(f, x)\right|^{1 / m+n+k} \geq M\right\} \in \mathcal{J} .
$$

Definition 2.10 It is said that a point $L \in X$ is an J-accumulation point and suppose that $f$ is a continuous function on $[0,1]$. A Bernstein polynomials $\left(B_{m n k}(f, x)\right)$ is a metric space $(X, d)$ iff for each $\epsilon>0$ the set

$$
\left\{(m, n, k) \in \mathbb{N}^{3}: d\left(B_{m n k}(f, x), f(x)\right)=\left|B_{m n k}(f, x)-f(x)\right|<\epsilon\right\} \notin \mathcal{J} .
$$


The set consisting of all J-accumulation points of $\left(B_{m n k}(f, x)\right)$ is symbolized by $\mathcal{J}\left(\Gamma\left(B_{m n k}(f, x)\right)\right)$.

Definition 2.11 Suppose that $f$ is a continuous function on $[0,1]$. It is said that a triple sequence of Bernstein polynomials $\left(B_{m n k}(f, x)\right)$ is rough J-convergent if J-LIM ${ }^{r} B_{m n k}(f, x) \neq \phi$. It is obvious that if $\mathcal{J}-\operatorname{LIM}^{r} B_{m n k}(f, x) \neq \phi$ for a triple sequence of Bernstein polynomials $\left(B_{m n k}(f, x)\right)$ of real numbers, under these conditions we conclude $\mathcal{J}-\operatorname{LIM}^{r} B_{m n k}(f, x)=$ [J-lim sup $\left.B_{m n k}(f, x)-r, \mathcal{J}-\lim \inf B_{m n k}(f, x)+r\right]$.

Definition 2.12 Assume that $f$ is a continuous function on [0,1]. It is said that a triple sequence of Bernstein polynomials $\left(B_{m n k}(f, x)\right)$ is rough J - core $B_{m n k}(f, x)$ is defined to the closed interval $[+\infty,-\infty]$.

\section{Main Results}

Theorem 3.1 Assume that $f$ is a continuous function on $[0,1]$. When a triple sequence of Bernstein polynomials $\left(B_{m n k}(f, x)\right)$ of real numbers and $\mathcal{J} \subset 2^{\mathbb{N}}$ be an admissible ideal, then we conclude that diam (J $\left.-\operatorname{LIM}^{r} B_{m n k}(f, x)\right) \leq 2 r$. Generally, diam $\left(\mathcal{J}-\operatorname{LIM}^{r} B_{m n k}(f, x)\right)$ has an upper bound.

Proof. Assume that diam $\left(\operatorname{LIM}^{r} B_{m n k}(f, x)\right)$. Then,

$$
\exists B_{m n k}(p, x), B_{m n k}(q, x) \in \operatorname{LIM} M^{r} B_{m n k}(f, x) \ni:\left|B_{m n k}(p, x)-B_{m n k}(q, x)\right|>2 r .
$$

Take $\epsilon \in\left(0, \frac{\left|B_{m n k}(p, x)-B_{m n k}(q, x)\right|}{2}-r\right)$. Because $B_{m n k}(p, x), B_{m n k}(q, x) \in \mathcal{J}-\operatorname{LIM}^{r} B_{m n k}(f, x)$, we have $A_{1}(\epsilon) \in \mathcal{J}$ and $A_{2}(\epsilon) \in \mathcal{J}$ for every $\epsilon>0$, where

$$
\begin{gathered}
A_{1}(\epsilon)=\left\{(i, j, k) \in \mathbb{N}^{3}:\left|B_{m n k}(f, x)-B_{m n k}(p, x)\right| \geq r+\epsilon\right\} \text { and } \\
A_{2}(\epsilon)=\left\{(i, j, k) \in \mathbb{N}^{3}:\left|B_{m n k}(f, x)-B_{m n k}(q, x)\right| \geq r+\epsilon\right\} .
\end{gathered}
$$

Using the properties $F(\mathcal{J})$, we get $\left(A_{1}(\epsilon)^{c} \cap A_{2}(\epsilon)^{c}\right) \in F(\mathcal{J})$. Thus we write,

$$
\begin{aligned}
\left|B_{m n k}(p, x)-B_{m n k}(q, x)\right| & \leq\left|B_{m n k}(f, x)-B_{m n k}(p, x)\right|+\left|B_{m n k}(f, x)-B_{m n k}(q, x)\right| \\
& <(r+\epsilon)+(r+\epsilon)<2(r+\epsilon), \text { for all }(m, n, k) \in A_{1}(\epsilon)^{c} \bigcap A_{2}(\epsilon)^{c}
\end{aligned}
$$

and this is an clear contradiction. Hence $\operatorname{diam}\left(\operatorname{LIM}^{r} B_{m n k}(f, x)\right) \leq 2 r$.

Now, consider a triple sequence of Bernstein polynomials of $\left(B_{m n k}(f, x)\right)$ of real numbers so that $\mathcal{J}-\lim _{m n k \rightarrow \infty} B_{m n k}(f, x)=f(x)$.

Let $\epsilon>0$. So, it can be written that $\left\{(m, n, k) \in \mathbb{N}^{3}:\left|B_{m n k}(f, x)-(f, x)\right| \geq \epsilon\right\} \in \mathcal{J}$. Consequently, we got

$$
\begin{aligned}
\left|B_{m n k}(f, x)-B_{m n k}(p, x)\right| & \leq\left|B_{m n k}(f, x)-(f, x)\right|+\left|(f, x)-B_{m n k}(p, x)\right| \\
& \leq\left|B_{m n k}(f, x)-(f, x)\right|+r \leq r+\epsilon
\end{aligned}
$$

for each $B_{m n k}(p, x) \in \bar{B}_{r}((f, x)):=\left\{B_{m n k}(p, x) \in \mathbb{R}^{3}:\left|B_{m n k}(p, x)-(f, x)\right| \leq r\right\}$. Then, we get $\left|B_{m n k}(f, x)-B_{m n k}(p, x)\right|<r+\epsilon$ for each $(m, n, k) \in\left\{(m, n, k) \in \mathbb{N}^{3}:\left|B_{m n k}(f, x)-(f, x)\right|<\epsilon\right\}$. Because the triple sequence of Bernstein polynomials of $B_{m n k}(f, x)$ is J-convergent to $f(x)$, we have

$$
\left\{(m, n, k) \in \mathbb{N}^{3}:\left|B_{m n k}(f, x)-(f, x)\right|<\epsilon\right\} \in F(\mathcal{J}) .
$$


Therefore, we get $p \in \mathcal{J}-\operatorname{LIM}^{r} B_{m n k}(f, x)$. Consequently, we can write

$$
\mathcal{J}-\operatorname{LIM}^{r} B_{m n k}(f, x)=\bar{B}_{r}((f, x)) .
$$

Since $\operatorname{diam}\left(\bar{B}_{r}((f, x))\right)=2 r$, this can be usually shown that the upper bound $2 r$ of the diameter of the set $\mathcal{J}-\operatorname{LIM}^{r} B_{m n k}(f, x)$ is not lower bound.

Theorem 3.2 Assume that $f$ is a continuous function prescribed on $[0,1]$. Assume that a triple sequence of Bernstein polynomials $\left(B_{m n k}(f, x)\right)$ of real numbers, J $\subset 3^{\mathbb{N}}$ is an admissible ideal. For a given arbitrary $(f, c) \in \mathcal{J}\left(\Gamma_{x}\right)$, we have $\left|B_{m n k}(f, x)-(f, c)\right| \leq r$ for every $B_{m n k}(f, x) \in \mathcal{J}-\operatorname{LIM}^{r} B_{m n k}(f, x)$.

Proof. If we suppose, on the contrariwise, that there is a point $(f, c) \in \mathcal{J}\left(\Gamma_{x}\right)$ and $B_{m n k}(f, x) \in$ $\mathcal{J}-\operatorname{LIM}^{r} B_{m n k}(f, x)$ so that $\left|B_{m n k}(f, x)-(f, c)\right|>r$. Define $\epsilon:=\frac{\left|B_{m n k}(f, x)-(f, c)\right|-r}{3}$. Then

$$
\left\{(m, n, k) \in \mathbb{N}^{3}:\left|B_{m n k}(f, x)-(f, c)\right|<\epsilon\right\} \subseteq\left\{(m, n, k) \in \mathbb{N}^{3}:\left|B_{m n k}(f, x)-(f, x)\right| \geq r+\epsilon\right\} .
$$

Since $(f, c) c \in \mathcal{J}\left(\Gamma_{x}\right)$, we have $\left\{(m, n, k) \in \mathbb{N}^{3}:\left|B_{m n k}(f, x)-(f, c)\right|<\epsilon\right\} \notin \mathcal{J}$. But from definition of J-convergence, since $\left\{(m, n, k) \in \mathbb{N}^{3}:\left|B_{m n k}(f, x)-f(x)\right| \geq r+\epsilon\right\} \in \mathcal{J}$, so by (3.2) we have $\left\{(m, n, k) \in \mathbb{N}^{3}:\left|B_{m n k}(f, x)-(f, c)\right|<\epsilon\right\} \in \mathcal{J}$, which contradicts the fact $(f, c) \in \mathcal{J}\left(\Gamma_{x}\right)$. On the way around, if $(f, c) \in \mathcal{J}\left(\Gamma_{x}\right)$ i.e., $\left\{(m, n, k) \in \mathbb{N}^{3}:\left|B_{m n k}(f, x)-(f, c)\right|<\epsilon\right\} \notin \mathcal{J}$, then $\left\{(m, n, k) \in \mathbb{N}^{3}:\left|B_{m n k}(f, x)-(f, x)\right| \geq r+\epsilon\right\} \notin \mathcal{J}$, which is an obvious contradiction to the fact $(f, x) \in \mathcal{J}-\operatorname{LIM}^{r} B_{m n k}(f, x)$.

Theorem 3.3 Assume that $f$ is a continuous function on [0,1]. A triple sequence of Bernstein polynomials

$$
\left(B_{m n k}(f, x)\right) \rightarrow^{\mathcal{J}}(f, x) \Leftrightarrow \mathcal{J}-\operatorname{LIM}^{r} B_{m n k}(f, x)=\bar{B}_{r}((f, x)) .
$$

Proof. Necessity: By Theorem 3.1.

Sufficiency: Let J $-\operatorname{LIM}^{r} B_{m n k}(f, x)=\bar{B}_{r}((f, x))(\neq \phi)$. Therefore the triple sequence spaces of Bernstein polynomials of $\left(B_{m n k}(f, x)\right)$ is J-analytic. Let us suppose that $(f, x)$ has got another J-cluster point $\left(f^{\prime}, x\right)$ which is differing from $(f, x)$. The point

$$
\begin{aligned}
(\bar{f}, x) & =(f, x)+\frac{r}{\left|(f, x)-\left(f^{\prime}, x\right)\right|}\left((f, x)-\left(f^{\prime}, x\right)\right) \\
(\bar{f}, x)-\left(f^{\prime}, x\right) & =(f, x)-\left(f^{\prime}, x\right) \\
+ & \frac{r}{\left|(f, x)-\left(f^{\prime}, x\right)\right|}\left[\left((f, x)-\left(f^{\prime}, x\right)\right)-\left(\left(f^{\prime}, x\right)-\left(f^{\prime}, x\right)\right)\right] \\
\left|(\bar{f}, x)-\left(f^{\prime}, x\right)\right| & =\left|(f, x)-\left(f^{\prime}, x\right)\right|+\frac{r}{\left|(f, x)-\left(f^{\prime}, x\right)\right|}\left|(f, x)-\left(f^{\prime}, x\right)\right| \\
\left|(\bar{f}, x)-\left(f^{\prime}, x\right)\right| & =\left|(f, x)-\left(f^{\prime}, x\right)\right|+r>r .
\end{aligned}
$$

Since $\left(f^{\prime}, x\right) \in \mathcal{J}\left(\Gamma_{x}\right)$, by Theorem 3.2, $(\bar{f}, x) \notin \mathcal{J}-L I M^{r} B_{m n k}(f, x)$. It is not possible as $|(\bar{f}, x)-(f, x)|=r$ and $\mathcal{J}-\operatorname{LIM}^{r} B_{m n k}(f, x)=\bar{B}_{r}((f, x))$. Because $(f, x)$ is the unique $\mathcal{J}$ cluster point of $(f, x)$. Hence $\Longrightarrow B_{m n k}(f, x) \rightarrow^{\mathcal{J}} f(x)$.

Corollary 3.1 If $(X,|.,|$.$) is a strictly convex spaces and let us assume that f$ is a continuous function defined on $[0,1]$. A triple sequence of Bernstein polynomials $\left(B_{m n k}(f, x)\right) \in X$, there exists $y_{1}, y_{2} \in \mathcal{J}-L I M^{r} B_{m n k}(f, x)$ so that $\left|y_{1}-y_{2}\right|=2 r$, then this triple sequence $(f, x) \rightarrow^{\mathcal{J}} \frac{y_{1}+y_{2}}{2}$. 
Theorem 3.4 If J $-L I M^{r} \neq \phi$, then J $-\lim \sup B_{m n k}(f, x)$ and J $-\liminf B_{m n k}(f, x)$ belong to the set J - LIM ${ }^{2 r} B_{m n k}(f, x)$.

Proof. We know that $\mathcal{J}-\operatorname{LIM}^{r} B_{m n k}(f, x) \neq \phi$, a triple sequence of Bernstein polynomials of $\left(B_{m n k}(f, x)\right)$ is J-analytic. The number J-liminf $B_{m n k}(f, x)$ is an J-cluster point of $(f, x)$ and consequently, we have $\left|(f, x)-\mathcal{J}-\liminf B_{m n k}(f, x)\right| \leq r \forall(f, x) \in \mathcal{J}-\operatorname{LIM}^{r}(f, x)$. Let $A=\left\{(m, n, k) \in \mathbb{N}^{3}:\left|(f, x)-B_{m n k}(f, x)\right| \geq r+\epsilon\right\}$. Now if $(m, n, k) \notin A$, then

$$
\left|B_{m n k}(f, x)-\left(\mathcal{J}-\liminf B_{m n k}(f, x)\right)\right| \leq\left|B_{m n k}(f, x)-(f, x)\right|+\left|(f, x)-\left(\mathcal{J}-\liminf B_{m n k}(f, x)\right)\right|<2 r+\epsilon .
$$

Thus $\mathcal{J}-\liminf B_{m n k}(f, x) \in \mathcal{J}-\operatorname{LIM}^{2 r} B_{m n k}(f, x)$. Likewise, it can be seen that $\mathcal{J}-$ $\lim \sup x_{m n k} \in \mathcal{J}-L^{2 r} x_{m n k}$.

Corollary 3.2 Assume that $f$ is a continuous function given on the prescribed interval [0,1]. A triple sequence of Bernstein polynomials $\left(B_{m n k}(f, x)\right)$ of real numbers, if J-LIMr $B_{m n k}(f, x) \neq$ $\phi$, then J-core $\{f(x)\} \subseteq \mathcal{J}-L I M^{2 r} B_{m n k}(f, x)$.

Proof.We now have J-LIM $\boldsymbol{M}^{r} B_{m n k}(f, x)=\left[\mathcal{J}-\limsup B_{m n k}(f, x)-2 r, \mathcal{J}-\liminf B_{m n k}(f, x)+2 r\right]$. Then the result is followed from Theorem 3.4.

Theorem 3.5 Assume that $f$ is a continuous function defined on [0,1]. A triple sequence of Bernstein polynomials $\left(B_{m n k}(f, x)\right)$ of real numbers. Under these conditions, the $\operatorname{diam}\left(\mathcal{J}-\operatorname{core}\left\{B_{m n k}(f, x)\right\}\right)$ of the set

$$
\mathcal{J}-\operatorname{core}\left\{B_{m n k}(f, x)\right\}=r \Longleftrightarrow \mathcal{J}-\operatorname{core}\{f(x)\}=\mathcal{J}-\operatorname{LIM}^{r} B_{m n k}(f, x) .
$$

Proof. $\operatorname{diam}\left(\mathcal{J}-\operatorname{core}\left\{B_{m n k}(f, x)\right\}\right)=r \Longleftrightarrow\left(\mathcal{J}-\lim \sup B_{m n k}(f, x)\right)-\left(\mathcal{J}-\liminf x_{m n k}\right)=r$

$$
\begin{aligned}
& \Longleftrightarrow \mathcal{J}-\operatorname{core}\left\{x_{m n k}\right\}=\left[\mathcal{J}-\liminf x_{m n k}, \mathcal{J}-\lim \sup B_{m n k}(f, x)\right] \\
&=\left[\mathcal{J}-\lim \sup B_{m n k}(f, x)-r, \mathcal{J}-\liminf B_{m n k}(f, x)+r\right]=\mathcal{J}-\operatorname{LIM}^{r} B_{m n k}(f, x) .
\end{aligned}
$$

And it can easily be seen that

(i) $r>\operatorname{diam}\left(\mathcal{J}-\operatorname{core}\left\{B_{m n k}(f, x)\right\}\right) \Longleftrightarrow \mathcal{J}-\operatorname{core}\left\{B_{m n k}(f, x)\right\} \subset \mathcal{J}-\operatorname{LIM}^{r} B_{m n k}(f, x)$,

(ii) $r<\operatorname{diam}\left(\mathcal{J}-\operatorname{core}\left\{B_{m n k}(f, x)\right\}\right) \Longleftrightarrow \mathcal{J}-\operatorname{LIM}^{r} B_{m n k}(f, x) \subset \mathcal{J}-\operatorname{core}\left\{B_{m n k}(f, x)\right\}$.

Theorem 3.6 Assume that $f$ is a continuous function defined on $[0,1]$. A triple sequence of Bernstein polynomials $\left(B_{m n k}(f, x)\right)$ of real numbers, if $\bar{r}=$ $\inf \left\{r \geq 0: \mathcal{J}-L_{I M} B_{m n k}(f, x) \neq \phi\right\}$, then $\bar{r}=\operatorname{radius}\left(\mathcal{J}-\operatorname{core}\left\{B_{m n k}(f, x)\right\}\right)$.

Proof. When the given set $\mathcal{J}-\operatorname{core}\left\{B_{m n k}(f, x)\right\}$ is singleton, then $\operatorname{radius}\left(\mathcal{J}-\operatorname{core}\left\{B_{m n k}(f, x)\right\}\right)=0$ and the triple sequence of Bernstein polynomials is J-convergent, i.e., J $-\operatorname{LIM}^{0} B_{m n k}(f, x) \neq \phi$. Therefore we obtain $\bar{r}=\operatorname{radius}\left(\mathcal{J}-\operatorname{core}\left\{B_{m n k}(f, x)\right\}\right)=0$.

Let us take the assumption that the set $\mathcal{J}-\operatorname{core}\left\{B_{m n k}(f, x)\right\}$ is now not a singleton. It can now be written as $\mathcal{J}-\operatorname{core}\left\{B_{m n k}(f, x)\right\}=[a, b]$ in which $a=\mathcal{J}-$ $\liminf B_{m n k}(f, x)$ and $b=\mathcal{J}-\limsup B_{m n k}(f, x)$. Again, let us suppose that $\bar{r} \neq$ radius $\left(\mathcal{J}-\operatorname{core}\left\{B_{m n k}(f, x)\right\}\right)$. When $\bar{r}<\operatorname{radius}\left(\mathcal{J}-\operatorname{core}\left\{x_{m n k}\right\}\right)$, we can define $\bar{\epsilon}=\frac{\frac{b-a}{2}-\bar{r}}{3}$. Now, be definition of $\bar{r}$ implies that $\mathcal{J}-L I M^{\bar{r}+\bar{\epsilon}} B_{m n k}(f, x) \neq \phi$, given $\epsilon>0 \exists l \in \mathbb{R}: A=$ $\left\{(m, n, k) \in \mathbb{N}:\left|B_{m n k}(f, x)-f(x)\right| \geq(\bar{r}+\bar{\epsilon})+\epsilon\right\} \in \mathcal{J}$. Since $\bar{r}+\bar{\epsilon}<\frac{b-a}{2}$ which is a contradiction of the definition of $a$ and $b$.

When $\bar{r}>\operatorname{radius}\left(\mathcal{J}-\operatorname{core}\left\{B_{m n k}(f, x)\right\}\right)$, then we can define $\bar{\epsilon}=\frac{\bar{r}-\frac{b-a}{2}}{3}$ and $r^{\prime}=\bar{r}-$ $2 \bar{\epsilon}$. It is obvious that $0 \leq r^{\prime} \leq \bar{r}$ and by definitions of $a$ and $b$, the number $\frac{b-a}{2} \in \mathcal{J}-$ $\operatorname{LIM}^{r^{\prime}} B_{m n k}(f, x)$. Under these conditions, we have $\bar{r} \in\left\{r \geq 0: \mathcal{J}-\operatorname{LIM}^{r} B_{m n k}(f, x) \neq \phi\right\}$, which is a contradiction to the equality $\bar{r}=\inf \left\{r \geq 0: \mathcal{J}-\operatorname{LIM}^{r} B_{m n k}(f, x) \neq \phi\right\}$ as $r^{\prime}<r$. 
Corollary 3.3 Assume that $f$ is a continuous function defined on [0,1]. A triple sequence of Bernstein polynomials $\left(B_{m n k}(f, x)\right)$ of real numbers, then $\mathcal{J}-$ core $\left\{B_{m n k}(f, x)\right\}=\mathcal{J}-$ $\operatorname{LIM}^{2 \bar{r}} B_{m n k}(f, x)$.

Example 3.1 Using the symbolic programming software Matlab, we have shown the comparisons and some easy-to-understand graphics for the convergence of operators given by (1.2) to the function $f(x)=1+x^{3} \sin (14 x)$ for various parameters. Looking at figure $1(a)$, it can be seen that as the $q$ and $p$ approaches towards 1 provided $0<q<p \leq 1,(p, q)$-BernsteinStancu operators presented by (1.2) converges towards the function $f(x)=1+x^{3} \sin (14 x)$. From figure $1(a)$ and $(b)$, it can be observed that for $\eta=\mu=0$, as the value the $(r, s, t)$ increases, $(p, q)$-Bernstein-Stancu operators (1.2) converges towards the function. Similarly from figure 2(a), it can be noticed that for $\eta=\mu=5$, as the value the $q$ and $p$ approaches towards 1 or some thing else provided $0<q<p \leq 1,(p, q)$-Bernstein-Stancu operators by (1.2) converges towards the function. From figure $2(a)$ and $(b)$, it is seen that when the value the $[r, s, t]$ increases, $(p, q)$-Bernstein-Stancu operators which are presented by $f(x)=1+x^{3} \sin (14 x)$ converges towards the function.

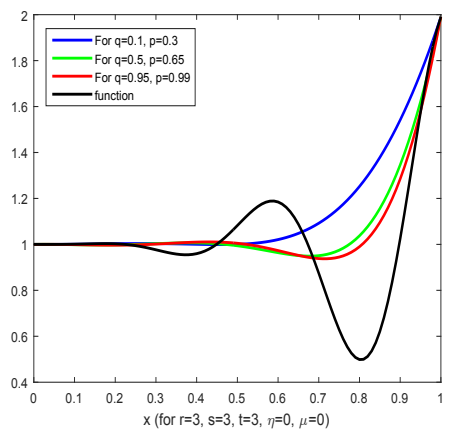

(a)

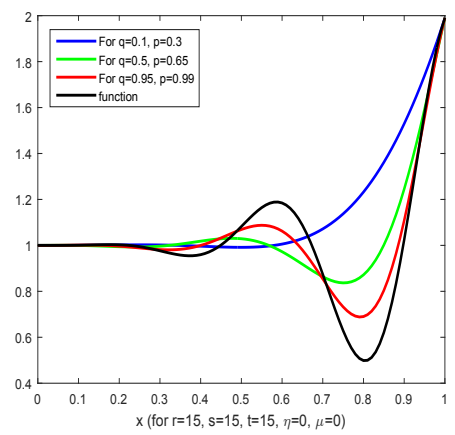

(b)

Figure 1: $(p, q)$-Bernstein-Stancu operators

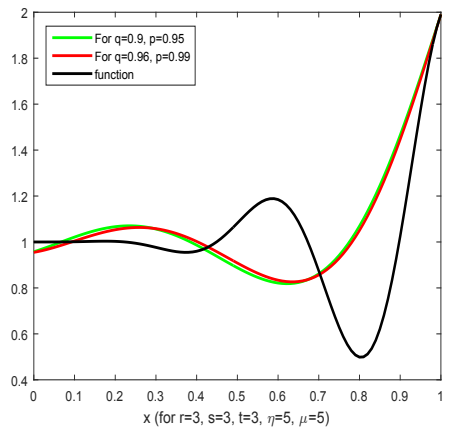

(a)

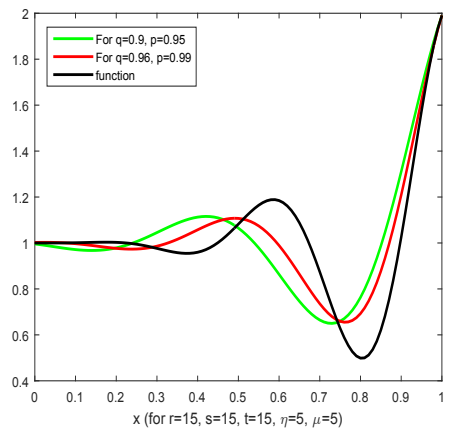

(b)

Figure 2: $(p, q)$-Bernstein-Stancu operators 
Competing Interests: We, the authors, hereby declare that there is not any conflict of interests in terms of the publication of this manuscript.

\section{References}

[1] Aytar S., Rough statistical convergence, Numer. Funct. Anal. Optim. 29(3-4), 291-303 (2008).

[2] Aytar S., The rough limit set and the core of a real sequence, Numer. Funct. Anal. Optim. 29(3-4), 283-290 (2008).

[3] Esi, A., On some triple almost lacunary sequence spaces defined by Orlicz functions, Research and Reviews: Discrete Mathematical Structures 1(2), 16-25 (2014).

[4] Esi, A. and Catalbas, M. N., Almost convergence of triple sequences, Global Journal of Mathematical Analysis 2(1), 6-10 (2014).

[5] Esi, A. and Savas, E., On lacunary statistically convergent triple sequences in probabilistic normed space, Appl. Math. Inf. Sci. 9(5), 2529-2534 (2015).

[6] Esi, A.; Araci, S. and Acikgoz, M., Statistical convergence of Bernstein operators, Appl. Math. Inf. Sci. 10(6), 2083-2086 (2016).

[7] Datta, A. J.; Esi, A. and Tripathy, B. C., Statistically convergent triple sequence spaces defined by Orlicz function, J. Math. Anal. 4(2), 16-22 (2013).

[8] Debnath, S.; Sarma, B. and Das, B. C., Some generalized triple sequence spaces of real numbers, J. Nonlinear Anal. Optim. 6(1), 71-79 (2015).

[9] Dundar, E.; Cakan, C., Rough J-convergence, Demonstr. Math. 47(3), 638-651 (2014).

[10] Phu, H. X., Rough convergence in normed linear spaces, Numer. Funct. Anal. Optim. 22(1-2), 199-222 (2001).

[11] Phu, H. X., Rough continuity of linear operators, Numer. Funct. Anal. Optim. 23(1-2), 139 - 146 (2002).

[12] Phu, H. X., Rough convergence in infinite dimensional normed spaces, Numer. Funct. Anal. Optimiz. 24(3-4), 285-301 (2003).

[13] Sahiner, A.; Gurdal, M. and Duden, F. K., Triple sequences and their statistical convergence, Selcuk J. Appl. Math. 8(2), 49-55 (2007).

[14] Sahiner, A.; Tripathy, B. C., Some J-related properties of triple sequences, Selcuk J. Appl. Math. 9(2), 9-18 (2008).

[15] Subramanian, N. and Esi, A., The generalized tripled difference of $\chi^{3}$ sequence spaces, Global Journal of Mathematical Analysis 3(2), 54-60 (2015). 